\title{
Aortic surgery in Loeys-Dietz syndrome: Excellent results, but close surveillance is necessary
}

\author{
John S. Ikonomidis, MD, PhD
}

\author{
From the Division of Cardiothoracic Surgery, Medical University of South Carolina, Charleston, SC. \\ Disclosures: Author has nothing to disclose with regard to commercial support. \\ Received for publication Nov 4, 2016; accepted for publication Nov 4, 2016; available ahead of print Dec 2, 2016. \\ Address for reprints: John S. Ikonomidis, MD, PhD, Division of Cardiothoracic Surgery, Suite BM 282, 114 \\ Doughty St, Charleston, SC 29425 (E-mail: ikonomij@musc.edu). \\ J Thorac Cardiovasc Surg 2017;153:413-4 \\ $0022-5223 / \$ 36.00$ \\ Copyright (c) 2016 by The American Association for Thoracic Surgery \\ http://dx.doi.org/10.1016/j.jtcvs.2016.11.010
}

Loeys-Dietz syndrome (LDS), first described in 2005, is an autosomal dominant connective tissue disorder characterized by generalized arterial tortuosity, hypertelorism, and a broad or bifid uvula or cleft palate. LDS was initially stratified into 2 types according to the severity of craniofacial features (type 1) or cutaneous features (type 2), but it is now classified by specific causative mutations in the transforming growth factor $\beta$ receptor (LDS types 1 and 2), mothers against decapentaplegic homolog 3 (LDS type 3), and transforming growth factor $\beta 2 \mathrm{~g}$ (LDS type 4) ligand genes, and the severities of the associated cardiovascular, craniofacial, cutaneous, and skeletal features are known to be gene mutation dependent.

The natural history of LDS is that of an overall aggressive connective tissue disorder that requires earlier than usual surgical intervention on the cardiovascular system where indicated. The types and results of these interventions are showcased in this month's issue of The Journal of Thoracic and Cardiovascular Surgery, in which Patel and colleagues ${ }^{2}$ present their series of 79 patients with LDS who underwent cardiovascular surgical management at Johns Hopkins University. These patients were young; the mean age at first operation was 25 years. Six patients were seen for acute aortic dissection. Five patients had a bicuspid aortic valve, and all had an ascending aortic aneurysm with a mean root diameter of $3.5 \mathrm{~cm}$. Twenty $(25 \%)$ patients had undergone previous sternotomy. Sixty-five (82\%) patients underwent aortic root replacement, 52 of whom underwent a valve-sparing operation and 4 of whom underwent a concomitant arch replacement. Nine patients overall $(11 \%)$ underwent aortic arch replacement, 2 (3\%) underwent isolated ascending aorta replacement, and 3 $(4 \%)$ underwent open thoracoabdominal repair. There were 2 operative deaths $(3 \%)$ and 8 late deaths. Nineteen patients underwent subsequent operations for late aneurysm or dissection. Mean follow-up was 6 years (range, 0-24 years). Kaplan-Meier survival was $88 \%$ at 10.73 years.

This is probably the largest single-center surgical report of this kind in the world. Excellent surgical results have

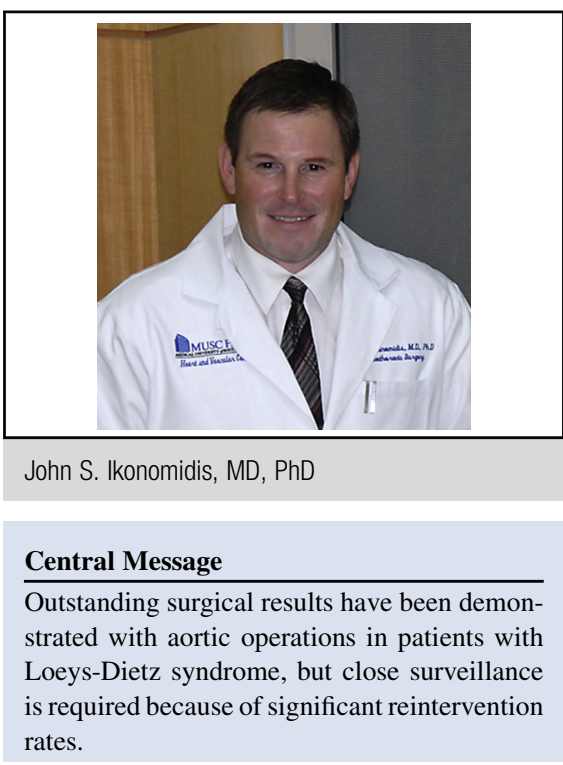

See Article page 406 .

been afforded by Dr Duke Cameron and his highly experienced surgical team at Johns Hopkins University. The report, which is well written and presented, highlights many issues specific to the management of these patients.

Genetic testing is critical in the diagnosis and management of aortic disease in patients with LDS, and surgical therapy is dictated to a significant extent by the aggressiveness of the known genotype. Whereas patients with Marfan syndrome show tendency toward aortic dissection when the aortic root diameter reaches $5 \mathrm{~cm}$, patients with LDS are at risk for dissection earlier, when the aortic root reaches $4 \mathrm{~cm}$. LDS types 1 and 2 are the most aggressive subtypes of LDS, and aortic root replacement is recommended at a threshold of $4 \mathrm{~cm}$, with specific mutations within this subgroup that argue in favor of surgery even earlier. LDS type 3 causes disease with intermediate severity, and aortic surgery is recommended at diameters of 4.0 to $4.5 \mathrm{~cm}$. LDS type 4 is the mildest form of the disease, and aortic root replacement is recommended at around $4.5 \mathrm{~cm}$. It is interesting that mean maximum aortic root diameter among the 65 patients with LDS who underwent aortic root replacement in this series was $4.17 \pm 0.97 \mathrm{~cm}$, the smallest root in this series was $2.0 \mathrm{~cm}$, and 21 of the 65 patients had root surgery with a maximum root diameter smaller than $4.0 \mathrm{~cm}$. This is a testament to the fact that surgical decision making in this population is dependent not just on the known genotype 
and aortic dimensions but also on the rate of growth, aortic valve function, severity of noncardiac phenotype, and family history.

Aortic operations on this patient population are associated with a higher incidence of reinterventions than those for degenerative aortic disease and Marfan syndrome. Overall, 19 patients $(24.6 \%)$ required reoperation in this series. Despite this, only 2 patients of the 52 who underwent valve-sparing root replacement required reoperation for valvular insufficiency. Given the fragile nature of the aortic and valvular tissues in these patients, these results are a testament to the outstanding surgical care provided by the Johns Hopkins University team, who point out that both technical and patient factors are important considerations for obtaining the best results. In the article of Patel and colleagues, ${ }^{2}$ it is stressed that the reimplantation technique is favored relative to the remodeling technique and is the procedure of choice to mitigate the development of late aortic insufficiency caused by annular dilatation. Accompanying this article is an excellent video describing the "Hopkins technique" for reimplantation valve-sparing aortic root replacement, which uses just 3 subannular sutures and encases the aortic valve in a Dacron polyester fabric graft with prefabricated sinuses of Valsalva. ${ }^{3}$ In addition, only patients with normal aortic valve cusps where considered for valve-sparing root replacement. Those patients whose valve cusps had large fenestrations and other abnormalities underwent composite valve-graft root replacement.
In the light of the significant aortic reintervention rate in these patients, close surveillance is critical. Patel and colleagues $^{2}$ recommend echocardiography every 3 to 6 months for the first year after surgery, which is then extended to every 6 months to 1 year. Full cardiovascular imaging should be obtained preoperatively and 1 year after surgical intervention, with reimaging at least every 2 years thereafter. It is further recommended that type B dissections be monitored particularly aggressively for aneurysm growth, with imaging at 7 to 14 days after dissection, repeated imaging at $1,3,6$, and 12 months, and yearly imaging thereafter.

To conclude, the article of Patel and colleagues ${ }^{2}$ describes the comprehensive, multidisciplinary, aggressive approach to the surgical treatment of aortic disease in patients with LDS. Because of the fragile nature of the vascular tissues inherent in this disorder, close surveillance is necessary, and it can be expected that about a quarter of the patients followed up will require surgical reintervention.

\section{References}

1. Van Laer L, Dietz H, Loeys B. Loeys-Dietz syndrome. Adv Exp Med Biol. 2014; 802:95-105.

2. Patel ND, Crawford T, Magruder JT, Alejo DE, Hibino N, Black J, et al. Cardiovascular operations for Loeys-Dietz syndrome: intermediate-term results. J Thorac Cardiovasc Surg. 2017;153:406-12.

3. Bethea BT, Fitton TP, Alejo DE, Barreiro CJ, Cattaneo SM, Dietz HC, et al. Results of aortic valve-sparing operations: experience with remodeling and reimplantation procedures in 65 patients. Ann Thorac Surg. 2004;78:767-72. 